\title{
Aortic aneurysm and diverticulum of Kommerell: a dreadful concomitance
}

\author{
Fernando Peixoto Ferraz de Campos ${ }^{a}$, Erasmo Simão da Silvab, \\ Brenda Margatho Ramos Martines ${ }^{c}$, João Augusto dos Santos Martines ${ }^{c}$
}

Campos FPF, Silva ES, Martines BMR, Martines JAS. Aortic aneurysm and diverticulum of Kommerell: a dreadful concomitance. Autopsy Case Rep [Internet]. 2012;2(4):45-51. http://dx.doi.org/10.4322/acr.2012.032

\section{ABSTRACT}

First described in 1936, the diverticulum of Kommerell (DOK) is a dilatation of the proximal segment of an aberrant subclavian artery. Appearing more frequently in the left-sided aortic arch, the aberrant right subclavian artery passes behind the esophagus toward the right arm, causing symptoms in the minority of cases. Diagnosis is generally incidental with this pattern. When symptomatic, dysphagia, respiratory symptoms, hoarseness, chest pain, and upper limb ischemia are the most common complaints. Although debatable, the origin of DOK is accepted as being degenerative or congenital. The degenerative condition is normally associated with atherosclerosis and occurs more frequently after the age of 50 years with no gender predominance. Complications may be life threatening and are more commonly related to the diverticulum aneurysm or when associated with aortic diseases such as aneurysms or dissection. The authors present a case of a 67-year-old male with a history of acute chest pain, neurological disturbances, and hypertensive crisis. The diagnostic workup revealed an aortic arch aneurysm with intramural hematoma and a diverticulum aneurysm of Kommerell. Treatment was conservative at first. The patient presented a satisfactory outcome and was referred to an outpatient clinic for follow up and further therapeutic consolidation.

Keywords: Aorta; Aneurysm; Dissection; Aberrant subclavian artery; Chest pain; Atherosclerosis.

\section{CASE REPORT}

A 67-year-old male patient, previously diagnosed with hypertension, diabetes mellitus, dyslipidemia and gout, was brought to the hospital with the history of acute interscapular pain followed by mental confusion and syncope. He denied dysphagia, or any respiratory symptoms, but lately noticed frequent choking. He was a heavy smoker until the age of 60 . He had never followed a regular treatment, and was hospitalized 16 years ago for a hypertensive crisis when an acute myocardial infarction was suspected. Recently, he was taking losartan, amlodipine, simvastatin, and gliclazide.

\footnotetext{
${ }^{a}$ Department of Internal Medicine - Hospital Universitário - Universidade de São Paulo, São Paulo/SP - Brazil.

${ }^{\text {b }}$ Department of Pathology - Faculdade de Medicina - Universidade de São Paulo, São Paulo/SP - Brazil.

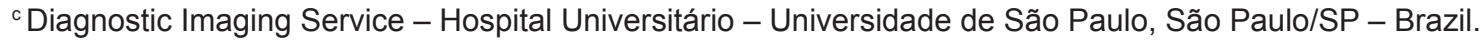

Copyright $\odot 2012$ Autopsy and Case Reports - This is an Open Access article distributed of terms of the Creative Commons Attribution NonCommercial License (http://creativecommons.org/licenses/by/3.0/) which permits unrestricted non-commercial use, distribution, and reproduction in any médium provided article is properly cited. 
The initial physical examination revealed an agitated patient, unconscious, with isochoric pupils, and normal and symmetric muscular strength. He was afebrile, hydrated but pale. His blood pressure was $230 / 146 \mathrm{mmHg}$ (symmetrical in both upper limbs), pulse rate was 124 beats per minute and regular, respiratory rate was 20 respiratory movements per minute, room air oximetry was $88 \%$, capillary blood glucose was $194 \mathrm{mg} / \mathrm{dL}$. The remaining physical examination was unremarkable.

With the diagnosis of hypertensive encephalopathy, the patient was treated with sodium nitroprusside, esmolol, morphine, and oxygen supplementation by Venturi mask. Gradual improvement of the level of consciousness and psychomotor agitation was observed. The initial laboratory tests are shown in Table 1.

The electrocardiogram showed sinus tachycardia with atrial extrasystoles and left ventricular hypertrophy. The chest radiography showed cardiomegaly with mediastinal enlargement (Figure 1). A brain CT scan was normal.

Due to the persistence of thoracic pain (which was also referred in the anterior area of the chest) and the image of mediastinal enlargement, the patient was submitted to a thoracic angiotomography that revealed a tortuous aorta with diffuse atheromatosis characterized by parietal thickening and calcified plaques. Dilatation of the aortic arch was evident, with a maximum diameter of $5.3 \mathrm{~cm}$. The presence of a spontaneous hyper density was observed along the upper posterior wall, displacing the intima and intimal calcification, which suggested intramural hematoma (Figure 2).
An aberrant right subclavian artery emerging from the posterior portion of the aortic arch passing behind and displacing the esophagus anteriorly and to the right is shown in Figure 3, presenting in its proximal segment a dilatation with $4.1 \mathrm{~cm}$ diameter characterizing a diverticulum aneurysm of Kommerell (Figures 3, 4 and 5).

The transesophageal echocardiography, performed at the bedside under sedation and topical anesthesia, revealed preserved ventricular systolic function, no segmental dysfunction of ventricular walls mobility, and thickening of the aortic and mitral valve rings with mild to moderate insufficiency. Aortic diameters were measured: aortic valve sinuses 41 $\mathrm{mm}$, tubular sinus junction $39 \mathrm{~mm}$, ascending aorta $53 \mathrm{~mm}$, and aortic arch $49 \mathrm{~mm}$.

The descending aorta showed a marked intimal thickening extending to the transition of the aortic arch, probably corresponding to a hematoma or thrombus. A mobile filament image in the

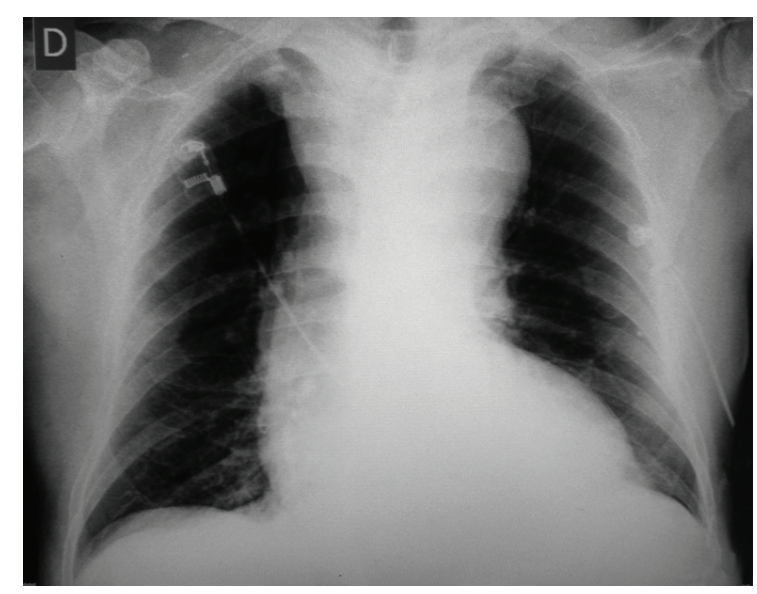

Figure 1 - Chest radiography showing enlarged cardiac image and mediastinal widening.

Table 1 - Initial laboratory examination workup

\begin{tabular}{cccccc}
\hline Exam & Result & $\mathbf{R V}$ & Exam & Result & RV \\
\hline Hemoglobin & 16.5 & $12.3-15.3 \mathrm{~g} / \mathrm{dL}$ & Creatinine & 1.3 & $0.4-1.3 \mathrm{mg} / \mathrm{dL}$ \\
Hematocrit & 48.9 & $36.0-45.0 \%$ & Urea & 57 & $10-50 \mathrm{mg} / \mathrm{dL}$ \\
Leukocytes & 12.1 & $4.4-11.3 .10^{3} / \mathrm{mm}^{3}$ & Ionized Ca+ & 1.22 & $1.15-1.35 \mathrm{Mmol} / \mathrm{L}$ \\
Bands & 0 & $1-5 \%$ & Sodium & 138 & $136-146 \mathrm{mEq} / \mathrm{L}$ \\
Segmented & 65 & $46-75 \%$ & Potassium & 4.1 & $3.5-5.0 \mathrm{mEq} / \mathrm{L}$ \\
Eosinophil & 3 & $1-4 \%$ & CKMB & 2.14 & $<5.0 \mathrm{ng} / \mathrm{mL}$ \\
Basophil & 1 & $0-2.5 \%$ & Troponin I & 0.04 & $<0.06 \mathrm{ng} / \mathrm{mL}$ \\
Lymphocytes & 26 & $18-40 \%$ & & & \\
Monocytes & 5 & $2-9 \%$ & & & \\
Platelets & 193 & $150-400.10^{3} / \mathrm{mm}^{3}$ & & & \\
\hline
\end{tabular}

$\mathrm{Ca}^{+}=$calcium $; \mathrm{CKMB}=$ creatine kinase $-\mathrm{MB} ; \mathrm{RV}=$ reference value . 

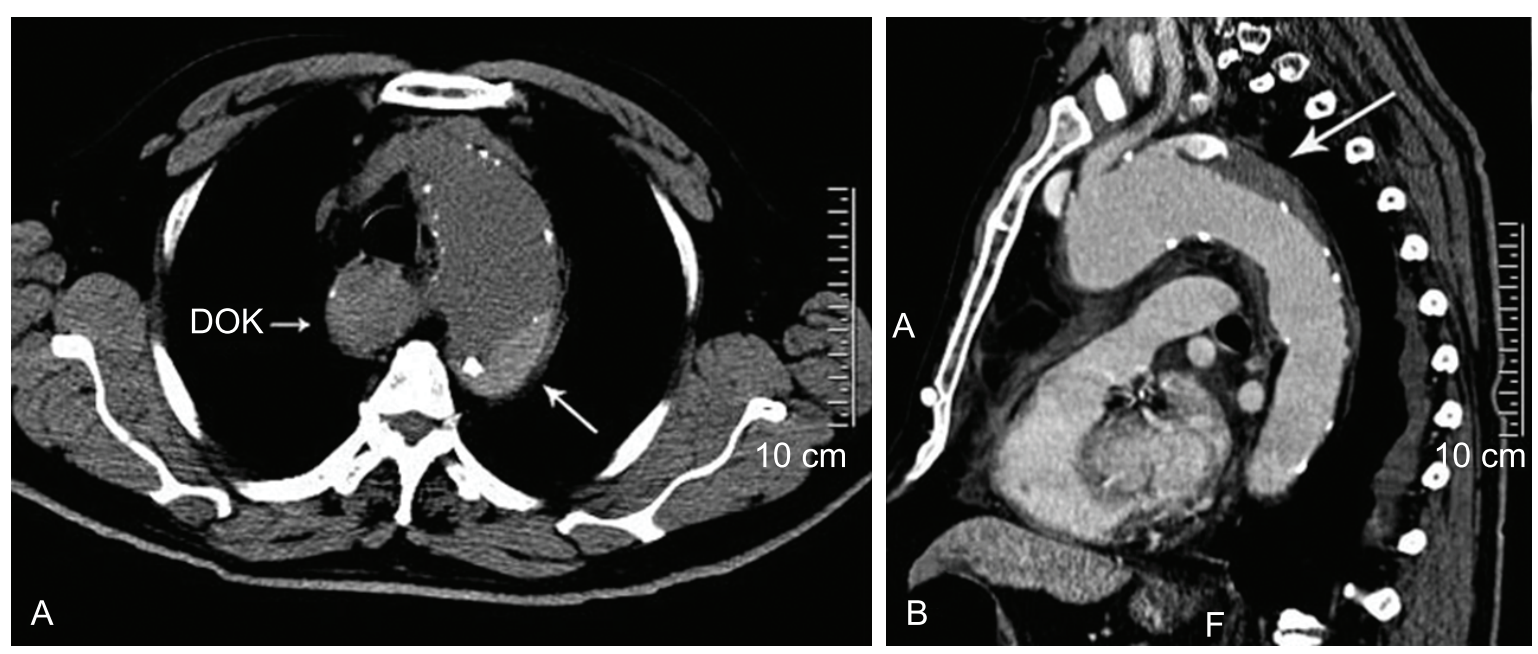

Figure 2 - A - Axial computed tomography of the thorax without intravenous contrast medium injection showing hyperdensity on the posterior wall of the aorta displacing the intima and its calcification, compatible with intramural hematoma; B - Multidetector CT - angiography of the thorax - Sagittal reformatted image showing intramural hematoma in the aortic arch, behind the intimal calcifications.
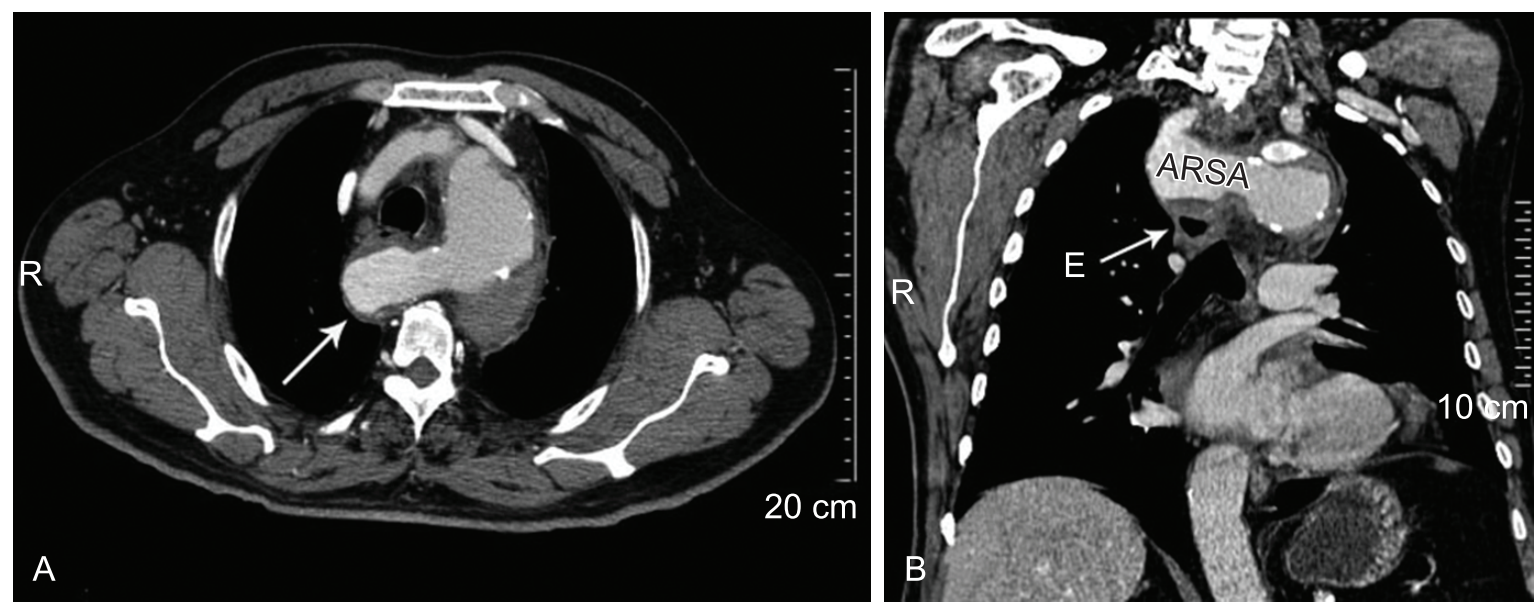

Figure 3-A - Axial computed angiotomography of the thorax after intravenous contrast medium injection showing dilatation and atheromatosis of the aberrant right subclavian artery (ARSA) (arrow); B - Multidetector CT - angiography of the thorax - Coronal reformatted image showing the ARSA and the displacement of the esophagus (arrow with E).
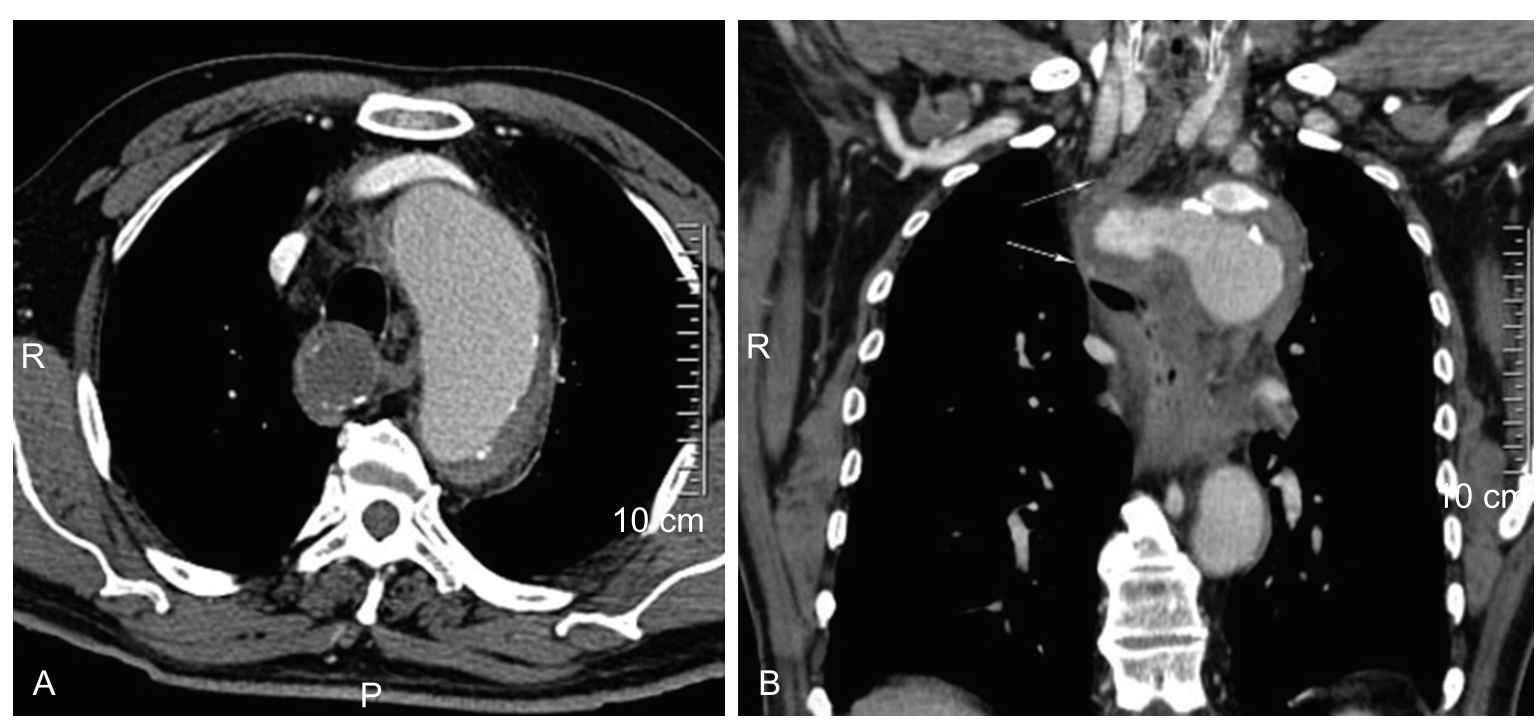

Figure 4 - A - Axial computed angiotomography of the thorax after intravenous contrast medium injection showing dilatation of the aberrant right subclavian artery with atheromatosis characterized by thickening and calcification of the vessel wall and mural thrombus; B - Note the compression and displacement of the esophagus (arrows). 

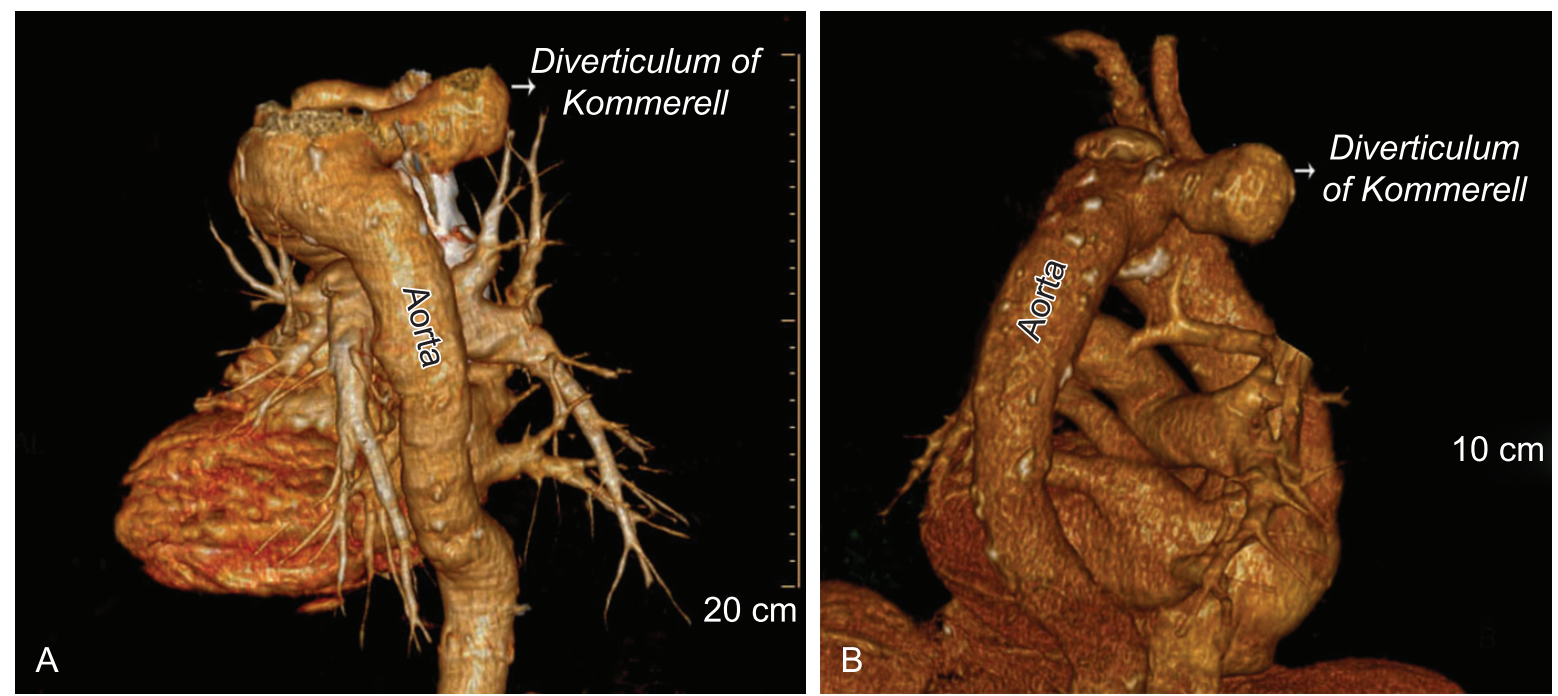

Figure 5 - A and B - Three-dimensional volumetric reconstruction of the posterior view of the aortic arch showing the diverticulum aneurysm of Kommerell in the emergence of the aberrant right subclavian artery.

descending aorta, suggestive of thrombus, was also evident.

The adopted treatment was conservative once the intramural thrombus remained steady and because of the unfeasibility for percutaneous treatment by the presence of the dilated ascendant aorta associated with the aberrant right subclavian artery.

The patient was discharged to outpatient follow up after 16 days of hospitalization.

\section{DISCUSSION}

Anomalous right subclavian artery has been described since 1735, but was first published 52 years later (1787) when David Bayford described the clinical symptom of dysphagia caused by the esophageal compression due to an aberrant right subclavian artery. ${ }^{1}$ He named the dysphagia as lusoria, from the Latin Lusus Naturae, which was kept up to the present. The English translation of Lusus Naturae means freak of nature. ${ }^{2}$ Aberrant subclavian artery (ASA) is one of the most common congenital anomalies of the aortic arch. The incidence of the aberrant right subclavian artery (ARSA) ranges from $0.4 \%$ to $2.3 \%$ in the general population and occurs in $37 \%$ of children with Down syndrome and congenital heart disease. ${ }^{3}$ Aberrant left subclavian artery (ALSA) is rare showing the incidence in only $0.05 \%$ of the general population. ${ }^{4}$
Burckhard Friedrich Kommerell, a German radiologist born in 1901 , described the first clinical diagnosis of an ASA. In 1936, examining a patient with a suspected diagnosis of stomach cancer he noticed a delayed passage of the barium contrast medium at the level of the aortic knob. In his report, he demonstrated that the esophagus was pushed and compressed forward. To the left and behind the esophagus there was a pulsatile mass construed to be a vessel. This finding was interpreted as being an aberrant origin of the right subclavian artery, and because of the size of the pulsatile mass it was attributed to an aortic diverticulum, from where the right subclavian artery originated. ${ }^{2}$ Since then the thoracic aortic diverticula were named after Kommerell. Nevertheless, the author called attention to the fact that an ASA does not always arise from a diverticulum (Figure 6 ).

The original Kommerell description comprised an aberrant artery arising from a left aortic arch. In this vascular anomaly, the right subclavian artery arises as the last branch of the aortic arch and courses from the proximal descending aorta to the right arm, passing behind the esophagus.

This anomaly generally courses without symptoms because of the lack of the trachea and esophagus encirclement by other vascular structures. This anomaly is associated with a diverticulum of Kommerell (DOK), in $60 \%$ of the cases. ${ }^{5,6}$ The newest edition of Rutherford's Vascular Surgery ${ }^{7}$ describes DOK as a degenerative aneurismal change in the proximal portion of the ASA or its aortic origin, keeping the controversy about the congenital or degenerative nature of DOK 


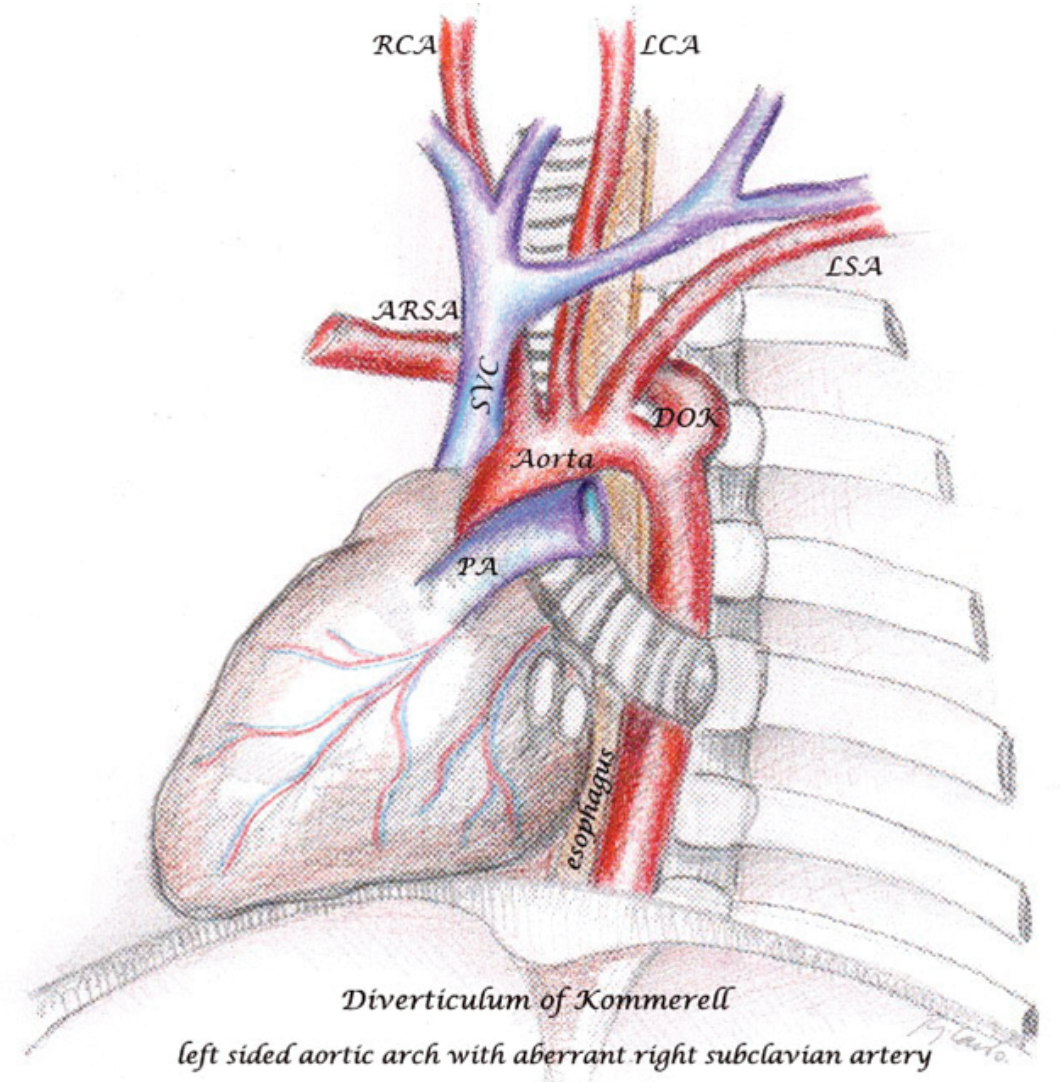

Figure 6 - Schematic left anterior oblique view of the left sided aortic arch with an aberrant right subclavian artery (ARSA) and a diverticulum of Kommerell (DOK). Note that the ARSA passes behind the esophagus. $\mathrm{LCA}=$ left carotid artery; LSA = left subclavian artery; PA = pulmonary artery; RCA = right carotid artery; $S V C=$ superior vena cava.

and how to distinguish it from a true aneurysm. ${ }^{4}$ Felson et al., addressed this difference of opinion well, dividing the entity into three categories: type 1 was the normal diverticulum described by Kommerell; type 2 was an aneurismal dilatation of this diverticulum; and type 3 was an aneurysm of the distal vessel separated from the diverticulum or aorta by a segment of normal caliber subclavian artery. ${ }^{8}$ However, he did not define the size of a "normal" diverticulum. Type 2 is called diverticulum aneurysm, and type 3 is the true ASA aneurysm. These two types are usually degenerative changes from atherosclerosis, which is most frequently encountered in adults older than 50 years of either sex. ${ }^{4,7}$ Type 1 DOK, a congenital enlargement, can be seen in both pediatric and adult patients. ${ }^{4}$ The patient of this report presented a DOK of $3.8 \mathrm{~cm}$ in diameter, which was most probably of degenerative atherosclerotic origin.

Since the description of Kommerell, attention was called to the fact that the subclavian artery does not always arise from a diverticulum, and symptoms are not always present. Indeed, most patients with ASA are asymptomatic and the diagnosis is usually made incidentally or when investigating aortic diseases nearby. ${ }^{4}$ Only $5 \%$ of patients with ARSA experience symptoms. Dysphagia of unclear etiology, also called dysphagia lusoria, is the most common presenting symptom. ${ }^{5}$ In children, a complete vascular ring formed by a ligamentum arteriosum or a ductus between the subclavian artery and the pulmonary artery compress the tracheobronchial tree and cause respiratory symptoms. Other reported symptoms are: dyspnea or coughing; chest pain; upper limb ischemia resulting from occlusive diseases, thromboembolism or rupture; and hoarseness due to compression of the recurrent laryngeal nerve. ${ }^{9}$ Symptoms are most apt to appear or to be aggravated (1) when the ASA is taut and stretches across the esophagus like a bow-string; (2) when the vessel becomes sclerotic and less elastic due to atherosclerosis; ${ }^{10,11}$ (3) when there is aneurysmal dilatation of the artery; ${ }^{12}$ or (4) when a vascular ring exists. The latter occurs when the right and left carotid arteries arise from the aortic arch together or in close proximity, so that the esophagus and trachea are hemmed in: dorsally by the aberrant right subclavian artery; and ventrally by the carotid fork. ${ }^{13}$ In the case of this report, the patient was poor of symptoms until recently when he referred frequent choking but not dysphagia. 
We interpreted this symptom as resulting from the compression of the esophagus by the ARSA because of the atherosclerotic hardening of the vessel and the size of the diverticulum aneurysm.

DOK may occur in other anomalies of the aorta arch system. In cases of right-sided aortic arch, which occurs in $0.5 \%$ of the general population, the ALSA occurs in $50 \%$ of these cases and arises from a diverticulum at the junction of the aortic arch and the right descending aorta. The ALSA passes obliquely upward behind the esophagus toward the left arm. ${ }^{5,6,14}$ The diverticulum occurs in $0.05-0.1 \%$ of these cases ${ }^{15,16}$ and is generally well developed because of the fetal ductus arteriosus at the junction of the aberrant artery..$^{10,11}$

Aneurysms arising in an ASA are truly rare ${ }^{17}$ and may constitute a potentially lethal condition, which is treatable when precociously identified. 6,18 . Complications of ASA aneurysms or DOK include: (1) rupture, occurring in 6-19\%; ;8-20 (2) embolization; ${ }^{14}$ (3) dissection; ${ }^{14}$ (4) upper gastrointestinal bleeding caused by a fistula involving the esophagus and the diverticulum; ${ }^{14}$ and (5) inadvertent puncture of the subclavian artery during central venous access (occurring in $3.7 \%$ of cases).

ASA may be associated with thoracic aortic aneurysm or aortic dissection. The aorta of patients with ASA is also abnormal and more prone to aneurysm formation, rupture, and dissection. ${ }^{4}$

Until 2005, 67 documented cases of ARSA were reported with DOK complicated by aneurysms, while ALSA with DOK were involved in only 22 cases. ${ }^{6}$ The majority of the aneurysms involving an ARSA with DOK are of atherosclerotic origin. These aneurysms may be involved with adjacent aortic dissection or rupture. In theses cases, the overall survival rate is less than $20 \%$. $^{17,18}$

A specific strategy for surgical treatment of DOK has not yet been established because of the variety on anatomical and morphological presentations. Various surgical and endovascular repairs have already been applied, such as total arch replacement, replacement of the descending aorta with ASA-carotid transposition, isolated stent graft, stent graft with extra-anatomic bypass, fenestrated and branched stented graft, and ligation of the ASA. ${ }^{15,19}$
Endovascular repair became the preferred intervention, but it depends on the anatomical evolvement of the ascendant aorta or aortic arch branches.

The patient of this case report presented to the hospital because of an aortic hematoma developed in a hypertensive crisis. The symptomatology started with interscapular pain that progressed to retrosternal pain, most probably because of the aortic dissection. He referred a recent choking, which we considered as a possible symptom of the ARSA with the diverticulum aneurysm, but this diagnosis was still considered as incidental. In this case, the adopted treatment was conservative, but the follow up will weight the necessity of surgical treatment. In the literature, surgical treatment for symptomatic DOK has shown good results when performed electively.

\section{REFERENCES}

1. Miller JM, Miller KS. A note on the historical aspects of dysphagia lusoria. Am Surg. 1992;58:502-3. PMid:1642389.

2. Van Son J, Konstantinov IE. Burckhard F. Kommerell and Kommerell's diverticulum. Tex Heart Inst J. 2002;29:109-12. PMid:12075866 PMCid:116736.

3. Kawashima A, Fishman EK, Kuhlman JE, Nixon MS. CT of posterior mediastinal masses. Radiographics. 1991;11:104567. PMid:1749849.

4. Yang C, Shu C, Li M, et al. Aberrant subclavian artery pathologies and Kommerell's diverticulum: A review and analysis of published endovascular/hybrid treatment options. J Endovasc Ther. 2012;19:373:82.

5. Brown DL, Chapman WC, Edwards, et al. Dysphagia lusoria: aberrant right subclavian artery with a Kommerell's diverticulum. Am Surg. 1993;59:583-6.

6. Fisher RG, Whigham CJ, Trinh C. Diverticula of Kommerell and aberrant subclavian arteries complicated by aneurysms. Cardiovasc Intervent Radiol. 2005;28:553-60. PMid:16091992. http://dx.doi.org/10.1007/s00270-003-0229-0

7. Timaran $\mathrm{CH}$. Upper extremity aneurysm. In: Rutherford JB, Cronenwett JL, Johnston W, editors. Rutherford vascular surgery. 7th ed. Philadelphia: WB Saunders; 2010. http:// dx.doi.org/10.1016/B978-1-4160-5223-4.00137-2

8. Felson B. Ruptured anomalous right subclavian artery: aneurysm or diverticulum? Semin Roentgenol. 1989;24:121-6. http://dx.doi.org/10.1016/0037-198X(89)90033-3 
9. Wu JY, Chen HY, Shu CC, et al. Kommerell diverticulum, right sided aorta, and left aberrant subclavian artery in a patient with dysphagia. J Thorac Cardiovasc Surg. 2010;139:e97-8. PMid:19660293. http://dx.doi.org/10.1016/j.jtcvs.2009.01.010

10. Backer CL, Ilbawi MN, Idriss FS, et al. Vascular anomalies causing tracheoesophageal compression. Review of experience in children. J Thorac Cardiovasc Surg. 1989;97:725-31. PMid:2651808.

11. Van Son JA, Julsrud PR, Hagler DJ, et al. Surgical treatment of vascular rings: the Mayo Clinic experience. Mayo Clinic Proc. 1993;68:1056-63. PMid:8231269.

12. Gross RE. Surgical treatment for dysphagia lusorian. Ann Surg. 1946;124:532-634. http://dx.doi.org/10.1097/00000658194609000-00008

13. Klinkhamer AC. Aberrant right subclavian artery. Clinical and roentgenologic aspects. Am J Roentgenol Radium Ther Nucl Med. 1966;97:438-46. PMid:5947142.

14. Agematsu K, Ueda T, Hoshino S, Nishiya Y. Rupture of Kommerell diverticulum after total arch replacement. Interact Cardiovasc Thorac Surg. 2012;11:800-2. PMid:20829386. http://dx.doi.org/10.1510/icvts.2010.241802
15. Ota T, Okada K, Takanashi S, Yamamoto S, Okita Y. Surgical treatment for Kommerell's diverticulum. J Thorac Cardiovasc Surg. 2006; 131:574-8. PMid:16515907. http:// dx.doi.org/10.1016/j.jtcvs.2005.10.012

16. Faucz RA, Furlan S, Barros AS, et al. Arco aórtico direito com artéria subclávia esquerda aberrante e divertículo de Kommerell. Radiol Bras. 2005;38:381-4. Portuguese. http:// dx.doi.org/10.1590/S0100-39842005000500014

17. Knight GC, Codd JE. Anomalous right subclavian artery aneurysms. Report of 3 cases, with a review of literature. Texas Heart Inst J. 1991;18:209-18. PMid:15227483 PMCid:325000.

18. Austin EH, Wolfe WG. Aneurysmal of aberrant subclavian artery with a review of literature. J Vasc Surg. 1985;2:571-7. PMid:3892054.

19. Cinà CS, Althani H, PasenauJ, Abouzahr L. Kommerell's diverticulum and right-sided aortic arch: a cohort study and review of literature. J Vasc Surg. 2004;2:571-7.

20. Kouchoukos NT, Masetti P. Aberrant subclavian artery and Kommerell aneurysm: surgical treatment with a standard approach. J Thorac Cardiovasc Surg. 2007;133:888-92. PMid:17382621. http://dx.doi.org/10.1016/j.jtcvs.2006.12.005

\section{Conflict of interest: None}

Submitted on: $11^{\text {th }}$ October 2012

Accept on: $8^{\text {th }}$ November 2012

Correspondence: Divisão de Clínica Médica

Av. Prof. Lineu Prestes, 2565 - Cidade Universitária - São Paulo/SP - Brazil

CEP: 05508-000 - Phone: +55 (11) 3091-9200

E-mail: ffcampos@usp.br 
